\title{
Long-term intake of saccharin decreases post-absortive energy expenditure at rest and is associated to greater weight gain relative to sucrose in wistar rats
}

\author{
Denise Entrudo Pinto', Kelly Carraro Foletto', Ramiro Barcos Nunes², Pedro Dal Lago² \\ and Marcello Casaccia Bertoluci ${ }^{1,3^{*}}$
}

\begin{abstract}
Background: Non-nutritive sweeteners (NNS) have been associated with increased prevalence of obesity. In previous studies, we demonstrated that saccharin could induce an increase in weight gain either when compared to sucrose or to a non-sweetened control at a similar total caloric intake. These data raised the hypothesis that reduced energy expenditure (EE) could be a potential mechanism explaining greater weight gain with saccharin use in rats. The aim of the present study was to compare long-term energy expenditure at rest between rats using saccharin or sucrose and correlate it with weight gain.

Methods: In the present study, we examine the potential impact of saccharin compared to sucrose in the EE of Wistar rats. In a controlled experiment of 17 weeks, 24 Wistar rats were divided into 2 groups: saccharin-sweetened yogurt (SAC) or sucrose-sweetened yogurt (SUC), plus a free chow diet. Only rats that consumed at least $70 \%$ of the offered yogurt were included. EE (kcal/day) was determined at rest through open circuit indirect calorimetry system in the early post-absorptive period with determinations of both $\mathrm{VO}_{2}$ consumption and $\mathrm{CO}_{2}$ production.

Measurements were evaluated at baseline, 5 and 12 weeks of dietary intervention. Weight gain, caloric intake (from yogurt, from chow and total) were determined weekly.

Results: Body weight and EE were similar between groups at baseline: $(p=.35)$ and $(p=.67)$ respectively. At the end of the study, SAC increased total weight gain significantly more in relation to SUC $(p=.03)$. Cumulative total caloric intake (yogurt plus chow) was similar between groups during the whole period $(p=.54)$. At 12 weeks, the EE was smaller in SAC compared to SUC $(p=.009)$. Considering both groups, there was a strong negative correlation between total weight gain and change in EE observed $[r(20)=-.61, p=.003]$. However, when analyzing the groups separately we found that SUC maintained this inverse correlation $[r(8)=-.68, p=.03]$, while SAC did not $[r(10)=-.33, p=.29]$.

Conclusion: These data support the hypothesis that long-term use of saccharin may blunt post-absorptive EE at rest in Wistar rats, which is related to weight gain. On the other hand, long-term sucrose intake can increase energy expenditure in rats. This effect combined can explain, at least partially, the weight gain increases associated to saccharin in relation to sucrose in these animals.
\end{abstract}

Keywords: Non-nutritive sweeteners, Saccharin, Sucrose, Weight gain, Energy expenditure, Oxygen consumption

\footnotetext{
*Correspondence: mcbertoluci@gmail.com

'Programa de Pós-Graduação em Medicina: Ciências Médicas, Universidade Federal do Rio Grande do Sul-UFRGS, Rua Ramiro Barcelos, n 2400, CEP 90035-003, Bairro Rio Branco, Porto Alegre, RS, Brazil

${ }^{3}$ Serviço de Medicina Interna, Hospital de Clínicas de Porto

Alegre-Universidade Federal do Rio Grande do Sul, Rua Ramiro Barcelos, $\mathrm{n}^{\circ}$

2350, CEP 90035-903, Bairro Rio Branco, Porto Alegre, RS, Brazil

Full list of author information is available at the end of the article
} 


\section{Background}

Non-nutritive sweeteners (NNS) have been associated with an increase in prevalence of obesity [1]. Epidemiological studies have shown that the use of NNS containing products correlates with the incidence of adiposity, metabolic syndrome (MS) [2, 3], type 2 diabetes mellitus (DM2) and cardiovascular disease [4, 5]. A clear cause and effect relationship, however, cannot be established.

The use of NNS, including saccharin, has been shown to interfere in the regulation of appetite and weight gain, which was demonstrated in some experimental studies and in randomized clinical trials [6-8]. Swithers and Davidson [9] conducted a series of experiments demonstrating that the use of saccharin resulted in reduced ability to compensate calories, providing a higher total caloric intake, greater weight gain and adiposity, when compared to the use of glucose.

In previous studies, we demonstrated that, in Wistar rats, saccharin can induce greater weight gain both in relation to sucrose [10] and to non-sweetened supplements [11], in spite of a similar total caloric intake. These experiments informed that, when rats receive saccharin-sweetened yogurt along with free chow intake, a compensatory increase in chow intake occurs. Nevertheless, the caloric deficit was perfectly compensated in a way that the total caloric intake (the sum of chow plus supplemented yogurt) was similar between groups. Despite this fact, significant increases in weight gain in the saccharin group could not be explained by increases in total caloric intake.

Mechanisms to explain why saccharin is associated with weight gain in rats are still not clear. One natural inference is to speculate that energy expenditure might be reduced in rats taking saccharin in relation to sucrose. Sprague-Dawley rats that were previously exposed to saccharin presented a smaller rise in the coretemperature after a standard meal when compared to rats pre-exposed to glucose, indicating that saccharin may interfere in heat production after meals and, therefore, in caloric expenditure [9].

In the present study, we hypothesized that the increase in long-term weight gain due to saccharin relatively to sucrose may be related to energy expenditure reduction induced by saccharin. We conducted a 17 -week experiment with Wistar rats consuming either saccharin or sucrose along with a free chow diet, and compared the energy expenditure (EE) between groups at rest, in the post-absortive period at baseline, 5 and 12 weeks.

\section{Methods}

\section{Study design and animals}

In a 17-week controlled experiment, 24 adult male Wistar rats weighing $\sim 200 \mathrm{~g}$ were randomly divided equally into 2 groups according to the type of sweetener: saccharin-sweetened yogurt (SAC group) or sucrosesweetened yogurt (SUC group), besides standard chow and water ad lib. Total weight gain and caloric intake were measured weekly. EE was measured at baseline, 5 weeks and 12 weeks. The researchers were blinded to the assigned groups.

Animals were individually housed in translucent polypropylene plastic cages, with controlled humidity $(65-70 \%)$ at room temperature $\left(22 \pm 1{ }^{\circ} \mathrm{C}\right)$, maintained through a 12 h-light and dark cycle.

Procedures were conducted in accordance with the Principles of Laboratory Animal Care (NIH publication No. 86-23, revised 1985) and guidelines of the National Research Council Committee for the Care and Use of Laboratory Animals [12] in the same degree as the Brazilian law for the scientific use of animals. Study protocol was approved by the ethics committee for experimental procedures of Universidade Federal de Ciências da Saúde de Porto Alegre (UFCSPA) and by the ethics committee for experimental procedures of Hospital de Clínicas de Porto Alegre (HCPA). This project was carried out in the Laboratory of Experimental Physiology at UFCSPA.

\section{Dietary manipulation}

All rats received standard chow pellets ad lib containing $2.93 \mathrm{kcal} / \mathrm{g}$, (Nuvital CR-1, Nuvilab ${ }^{\mathrm{nt}}$ ). Additional chow was added to the top of the cages every $24 \mathrm{~h}$ as needed, and chow rest was weighed and registered once a week. For weighing, all chow was removed and weighed with an electronic precision scale (AS 5500, Marte ${ }^{\mathrm{Tm}}$, SP, Brazil). The intake was calculated and registered weekly. The largest solid pellets were allocated in the grid feeders. A crumb collector was installed on the outside of the cage to minimize losses. Cages were carefully monitored for any evidence of chow spillage and crumbs were considered for the control of chow intake.

Sweetened yogurt supplements were prepared according to our former protocol $[10,11]$. In short, it included $20 \mathrm{ml}$ of standardized low-fat yogurt (Nestlé ${ }^{\mathrm{m}}{ }^{\mathrm{m}}$, SP, Brazil - containing per unit of $160 \mathrm{~g} 57 \mathrm{kcal}, 7.8$ grams of carbohydrate, 5.8 grams of protein and 0 grams of total fat) to which it was added either dry sucrose (União ${ }^{\mathrm{Tm}}$, SP, Brazil) or sodium-saccharin (Zero-Cal ${ }^{\mathrm{m}}$, SP, Brazil) to include $20 \%$ sucrose solution and $.3 \%$ saccharin solutions respectively. Yogurt supplements were offered $22 \mathrm{~h}$ daily, from 11 AM to 9 AM, 7 days a week during the whole experiment. Yogurt was offered in special bottles with adapted beaks to avoid leakage. Additionally, $15 \mathrm{ml}$ of pure water was added into yogurt to dilute and adjust viscosity in order to allow easier drinking. Caloric densities of sucrose-sweetened yogurt and saccharinsweetened yogurt were: $.63 \mathrm{kcal} / \mathrm{ml}(\sim 170 \mathrm{kcal} / \mathrm{wk})$ and 
$.24 \mathrm{kcal} / \mathrm{ml}(\sim 60 \mathrm{kcal} / \mathrm{wk})$, respectively. Rats that drank less than $70 \%$ of sweetened yogurt were excluded from the study. The yogurt bottles were also checked for any sign of leaking or clogging.

\section{Caloric intake determination}

Mean caloric intake was determined by the week difference between the weight of offered chow and the weight of the chow left in the grid, corrected by the rat weight at the end of the week using the following formula:

$$
\begin{aligned}
& \text { Mean Caloric Intake of } \text { Yogurt }_{1-17 w k}(\mathrm{kcal} / \mathrm{g} / \mathrm{wk}) \\
& =\left(\frac{\sum \mathrm{kcal} \mathrm{of} \mathrm{yogurt}_{1 \mathrm{wk}}}{\text { weight }_{1 \mathrm{wk}}}+\cdots+\frac{\sum \mathrm{kcal}_{\text {of yogurt }} \text { y }_{17 \mathrm{wk}}}{\text { weight }_{17 \mathrm{wk}}}\right) \\
& \div 17 \text { wk } \\
& \text { Mean Caloric Intake of Chow }{ }_{1-17 w k}(\mathrm{kcal} / \mathrm{g} / \mathrm{wk}) \\
& =\left(\frac{\sum \mathrm{kcal} \mathrm{of} \mathrm{chow}_{1 \mathrm{wk}}}{\text { weight }_{1 \mathrm{wk}}}+\cdots+\frac{\sum \mathrm{kcal} \mathrm{of} \mathrm{chow}_{17 \mathrm{wk}}}{\text { weight }_{17 \mathrm{wk}}}\right) \\
& \div 17 \mathrm{wk} \\
& \text { Mean Total Caloric Intake } 1-17 \mathrm{wk}(\mathrm{kcal} / \mathrm{g} / \mathrm{wk}) \\
& =\left(\frac{\sum \text { kcalf total }_{1 \mathrm{wk}}}{\text { weight }_{1 \mathrm{wk}}}+\cdots+\frac{\sum \mathrm{kcal} \mathrm{total}_{17 \mathrm{wk}}}{\text { weight }_{17 \mathrm{wk}}}\right) \\
& \div 17 \mathrm{wk}
\end{aligned}
$$

Cumulative total caloric intake (including yogurt and chow), cumulative caloric intake of yogurt and cumulative caloric intake of chow were calculated by the sum of calories ingested along each week, and corrected by the corresponding rat weight at the end of each week. These data were calculated in the cumulative mode for the 17-week period and were expressed as $\mathrm{kcal} / \mathrm{g}$ of rat.

\section{Body weight}

Rats were weighed weekly at the same time in the morning, using an electronic precision scale, suitable for weighing animals in motion (AS 5500, Marte ${ }^{\mathrm{Tx}}$, SP, Brazil). The absolute weight gain after 17 weeks of dietary intervention was calculated as follows:

Total weight gain $(\mathrm{g})=$ body weight $_{17 \mathrm{wk}}$ - body weight baseline

Cumulative weight gain was calculated by the subtraction of the basal weight from the weight obtained every week, and expressed in grams.

\section{Indirect calorimetric}

The protocol of indirect calorimetric was adapted from Rodrigues et al. [13]. Measurements were made using an open-system oxygen gas chamber, at controlled room temperature of $21{ }^{\circ} \mathrm{C}$. The metabolic chamber was connected to a vacuum pump that generates $2.5 \mathrm{~L} / \mathrm{min} \mathrm{O}_{2}$ flow. Oxygen flow was determined using $\mathrm{O}_{2}$ and $\mathrm{CO}_{2}$ analyzer
(AVS Projects ${ }^{\mathrm{TM}}$, São Carlos, SP, Brazil). Animals were placed into a gas chamber for 15 min to allow stabilization. After that, data started to be recorded for $5 \mathrm{~min}$.

Tests were performed 2-3 $\mathrm{h}$ in the post-absorptive period at rest in the baseline, week 5 and week 12. All chow and yogurt were removed $2 \mathrm{~h}$ before experiments. All tests were performed in duplicate, being repeated in the immediate next following day. The mean of 2 results was considered for statistical analysis.

\section{Determination of respiratory variables Oxygen Consumption $\left(\mathrm{VO}_{2}\right)$}

$\mathrm{VO}_{2}$ was calculated according to the flow of environment air pumped into the metabolic chamber using the formula:

$$
\mathrm{VO}_{2}\left(\mathrm{ml} / \mathrm{kg}^{-1} / \mathrm{min}^{-1}\right)=\mathrm{PF} \frac{(\mathrm{A}-\mathrm{E})}{\overline{\mathrm{BW}}}
$$

Where:

"PF" is the measured flow through the metabolic chamber $(2500 \mathrm{ml} / \mathrm{min})$;

"A" is the fraction of oxygen entering the chamber (environment air);

"E" is the fraction of effluent oxygen;

"BW" is the animal body weight in grams.

\section{Carbone Dioxide Production $\left(\mathrm{VCO}_{2}\right)$}

The $\mathrm{VCO}_{2}$ production determination was obtained from the carbon dioxide removal detected by a sensor at the chamber output expressed in $\mathrm{ml} / \mathrm{kg} / \mathrm{min}$.

Both $\mathrm{VO}_{2}$ and $\mathrm{VCO}_{2}$ were calculated according to ACQUAD software.

\section{Respiratory Exchange Ratio (RER)}

The RER, also known as respiratory quotient, was calculated according to the formula:

$$
\mathrm{RER}=\frac{\mathrm{VCO}_{2}}{\mathrm{VO}_{2}}
$$

\section{Energy Expenditure (EE)}

EE was estimated using the Weir equation [14] extrapolated to $24-\mathrm{h}$ :

$$
\mathrm{EE}(\mathrm{kcal} / \text { day })=\left(3.94 \mathrm{VO}_{2}+1.11 \mathrm{VCO}_{2}\right) \times 1440
$$

Where $\mathrm{VO}_{2}$ and $\mathrm{VCO}_{2}$ were expressed in $\mathrm{L} / \mathrm{min}$ and 1440 is the number of minutes in a day.

The change in EE was determined as the difference in $\mathrm{EE}$ at the end of 12 week and at baseline, calculated according to the formula:

$$
\text { Change in } \mathrm{EE}(\mathrm{kcal} / \text { day })=\mathrm{EE}_{12 \mathrm{wk}}-\mathrm{EE}_{\text {baseline }}
$$




\section{Statistical analysis}

The variables: total body weight gain, the area under the curve (AUC), mean caloric intake of chow, yogurt, and total caloric intake (chow plus sweetened-yogurt) were compared using $t$-tests.

ANOVA of repeated measures was used to compare the effect over time of cumulative weight gain, cumulative caloric intake (of sweetened-yogurt, of chow and total), RER, $\mathrm{VO}_{2}, \mathrm{VCO}_{2}$ and EE.

We used Pearson's correlation coefficient ( $r$ ) to evaluate the association between weight gain and change in energy expenditure over the entire experiment and it was presented graphically and numerically with $\mathrm{r}$ and $p$ values. Reported values are mean and standard error \pm $\mathrm{SE}$, and $p<.05$ was taken as significant for all analysis. Statistical analyses were performed using SPSS $^{\text {mi }} 21$ (IBM Coorporation, Armonk, NY, USA). AUCs were obtained through software NCSS 2007 (Number Cruncher Statistical Systems, Kaysville, UT, USA), calculated by the trapezoidal method.

\section{Results}

\section{Total weight gain}

At baseline, body weight (g) did not differ between groups: SUC: $219.00 \pm 6.74$ vs SAC: $207.33 \pm 9.48$ ); $t(20)=.96, p=.35$. After 17 weeks, SAC showed greater weight gain compared to SUC, respectively: $146.25 \pm 10.30$ vs $105.70 \pm 14.62 ; t(20)=-2.32, p=.03$. This corresponded to an increase from baseline of $71 \%$ and $48 \%$ in SAC and SUC, respectively. When we analyzed the weight gain of each week, we verified that the statistical significance occurred from the 16th week $[F(3.01,60.26)=2.99, p=.038]($ Fig. 1) .

\section{Caloric intake}

\section{Sweetened-yogurt intake}

Calories derived from sweetened-yogurt in SUC were 2.8 times greater than in SAC due to the higher caloric density in sucrose-sweetened yogurt; $t(9.87)=18.10, p<.001$.

The longitudinal and cumulative analysis showed statistically difference from the first week $[F(1.13,22.61)=$ 287.64, $p<.001$ ] (Fig. 2a). Two rats were excluded from the SUC group due to yogurt intake less than $70 \%$. There were no differences between groups in yogurt acceptance in the remaining rats, $(80 \% \pm .87) ; t(20)=-1.54, p=.14$.

\section{Chow intake}

The mean caloric intake from chow tended to be greater in SAC than in SUC, corresponding to a $10 \%$ compensatory increment, although not statistically significant; $t(20)=-1.56, p=.13$. In the repeated measure analysis, the time $\mathrm{x}$ group interaction was not significant $[F(1.42,28.44)=1.70, p=.21$. (Fig. $2 b$ ).

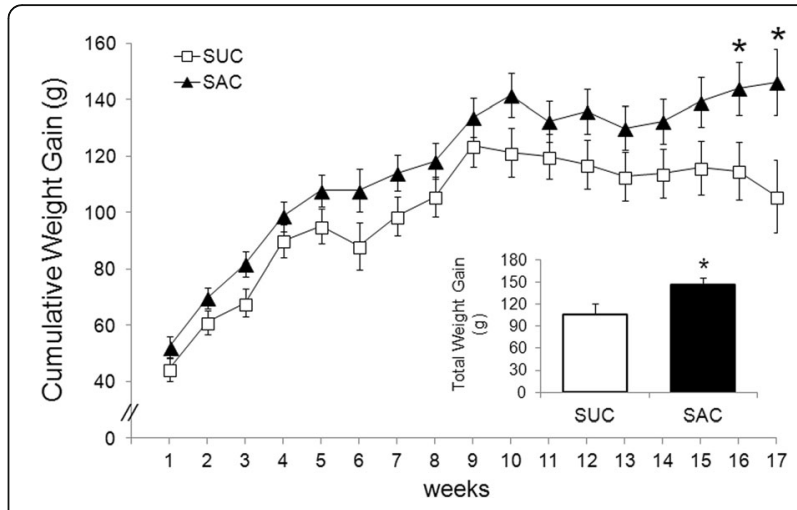

Fig. 1 Cumulative weight gain (g) over 17 weeks, determined by ANOVA of repeated measures. There was statistical difference from the 16th weeks $[F(3.01,60.26)=2.99, p=.038]$. Bar chart indicate analysis by Student's $t$-test [t(20) $=-2.32, p=.03]$. Group labels: SAC (Saccharin, $n=12$ ) and SUC (Sucrose, $n=10$ ). Error bars indicate SE and asterisk (*) indicates $p<.05$

\section{Total caloric intake}

Mean and Cumulative total caloric intake (chow intake plus sweetened-yogurt intake) was similar between groups in both analysis; $t(20)=.63, p=.54$ and $F(1.38,27.64)=$ $1.05, p=.34$, respectively. This result indicates that chow intake in SAC was perfectly compensatory to the lack of calories in saccharin-sweetened yogurt (Fig. 2c).

\section{Respiratory variables \\ $\mathrm{VO}_{2}$ consumption}

At baseline, 5th and 12th weeks, the $\mathrm{VO}_{2}$ consumption was similar between groups; $F(1,20.01)=.13, p=.72$. Further analysis showed that both groups, not differing from baseline. The analysis of $\mathrm{AUC}_{(0-12 \mathrm{wk})}$ between groups was also similar; $t(20)=.43, p=.67$ (Fig. 3a).

\section{$\mathrm{VCO}_{2}$ production}

At baseline, $\mathrm{VCO}_{2}$ production was similar between groups. At weeks 5 and $12, \mathrm{VCO}_{2}$ production was significantly greater in SUC group $F(1.42,28.39)=14.92$, $p<.001$. $\mathrm{VCO}_{2}$ increased progressively from baseline in SUC at weeks 5 and 12 respectively: $23 \%(p<.001)$ and $39 \%(p<.001)$, while there was no difference from baseline in SAC group. The area under the curve $\left(\mathrm{AUC}_{(0-12 \mathrm{wk})}\right)$ was $42 \%$ greater in $\mathrm{SUC}$ group $t(20)=6.86, p<.001$ (Fig. 3b).

\section{Respiratory Exchange Ratio (RER)}

At baseline, RER was similar between groups. At weeks 5 and 12 it was significantly lower in SAC than in SUC ( $p=.036$ and $p<.001$, respectively); it was also confirmed when we analyzed $\mathrm{AUC}_{(0-12 \mathrm{wk})} ; t(20)=$ 2.82, $p=.011$.

When we compared week 12 from baseline there was an increase in $29 \%$ on SUC and a decrease in 


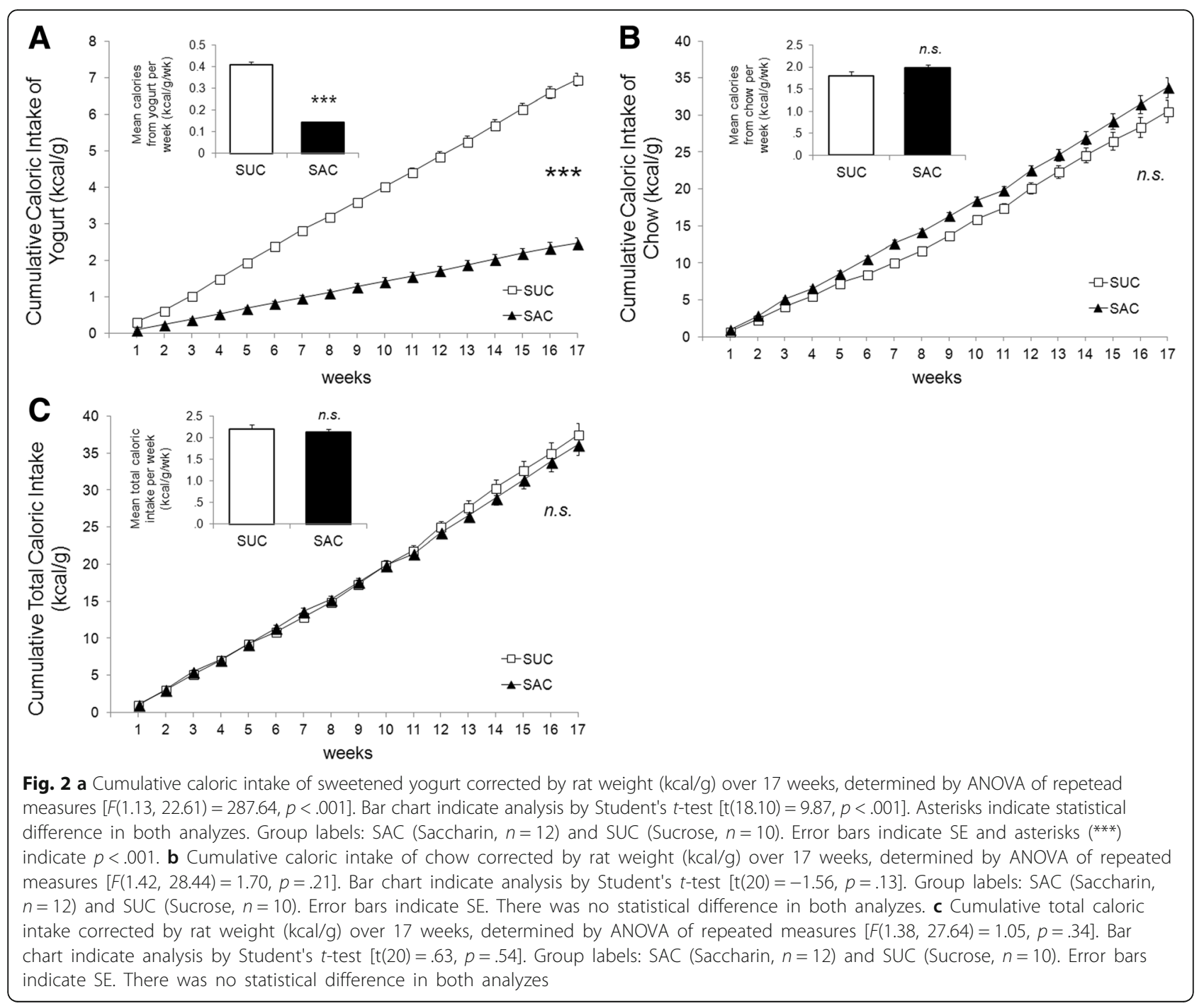

$15 \%$ on SAC, but without statistical difference $(p=.06$ and $p=.67$, respectively) (Fig. $3 \mathrm{c}$ ).

\section{Energy Expenditure (EE)}

At baseline there was no difference in respect of $\mathrm{EE}$ between groups: SUC: $194.66 \pm 14.95$ vs SAC: 185.92 $\pm 13.65 \mathrm{kcal} /$ day $(p=.67)$. At week 12 (maximum difference peak), EE was $16 \%$ greater in SUC than in SAC group $(p=.009)$ : SUC: $209.42 \pm 7.49$ vs SAC: $179.82 \pm 6.84 \mathrm{kcal} /$ day. In relation to baseline, SUC increased $\mathrm{EE}$ in $8 \%(p<.001)$ while SAC decrease $\mathrm{EE}$ in $3 \%(p=.04)$ (Fig. $3 \mathrm{~d})$.

\section{Correlation between Total Weight Gain and Change in Energy Expenditure (EE)}

Considering both groups together, there was a strong and negative correlation $[r(20)=-.61, p=.003]$ between total weight gain and the change (delta) in energy expenditure from baseline to 12 weeks. However when analyzing the groups separately, we found that SUC maintained a strong and significant inverse correlation $[r(8)=-.68, p=.03]$, while SAC did not $[r(10)=-.33, p=.29]$ (Fig. 4).

\section{Discussion}

The present study confirmed our former observations $[10,11]$ that rats consuming saccharin-sweetened yogurt had greater cumulative weight gain compared to rats eating sucrose-sweetened yogurt, when chow intake is not limited. Nonetheless, this increase was not related to differences in caloric intake, but rather to differences in EE between groups.

We observed that EE increased significantly $(p<.001)$ from baseline in rats eating SUC, and decreased slightly $(p=.04)$ in rats eating SAC, causing a significant difference between groups at 12 week $(p=.009)$. This difference was primarily determined by a significant increase in carbon dioxide production $\left(\mathrm{VCO}_{2}\right)$ in SUC group 


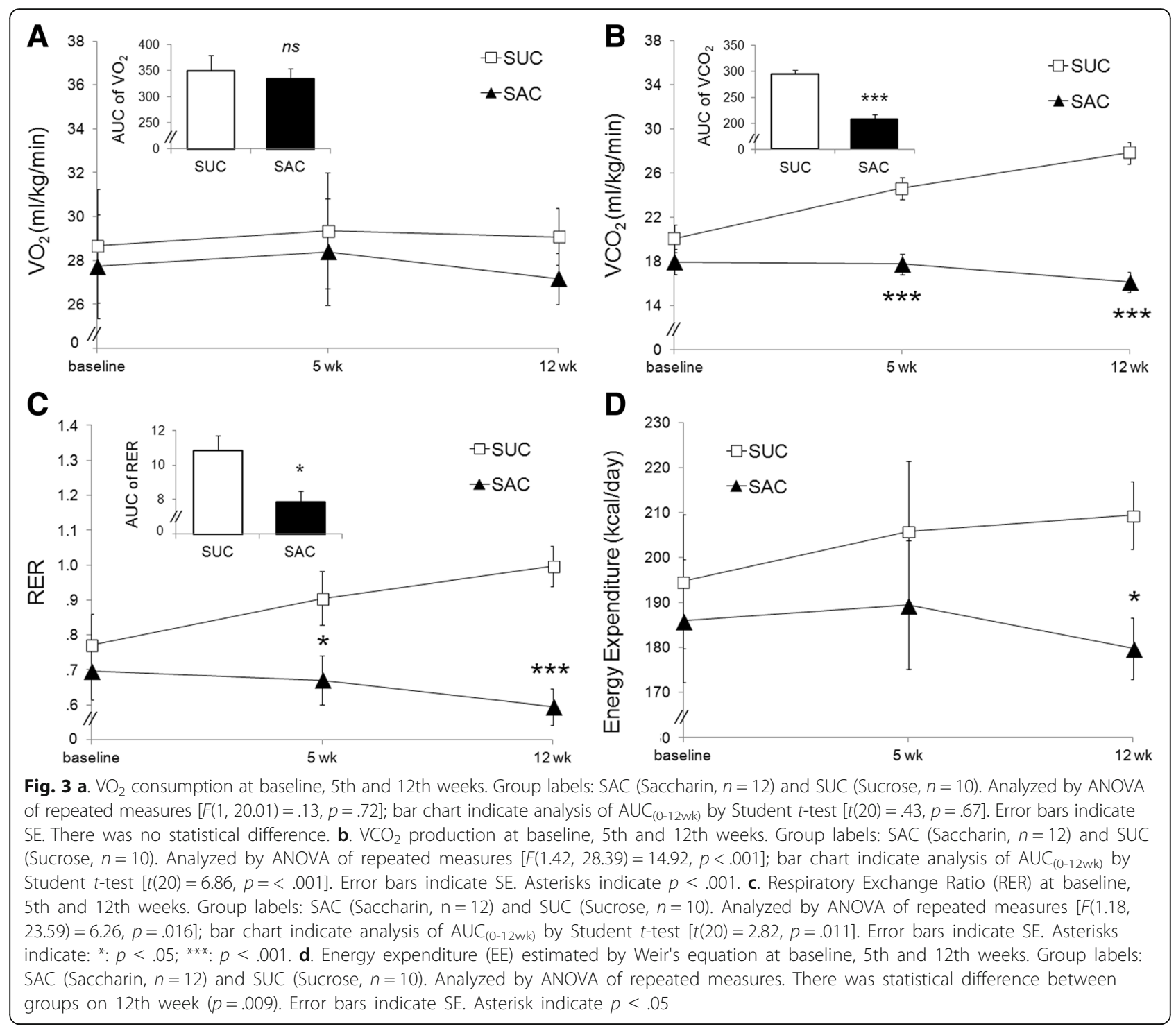

at weeks 5 and 12 from baseline, combined with a stable oxygen consumption $\left(\mathrm{VO}_{2}\right)$ in both groups. The change in EE (difference from week 12 to week 0 ), presented as a strong and inverse correlation with the rate of weight gain, which was observed in SUC group $(p=.03)$ but not in SAC group $(p=.29)$. This suggests that increases in EE could explain the observed differences in weight gain between groups.

As rats were tested in the early post-absorptive period, an increase in EE was expected to be due to the nutrient absorption and digestive metabolism. So, it was interesting that, in contrast to SUC, EE in SAC group did not rise. This effect could be explained to either a lack of carbohydrate intake in SAC group or to a possible direct blunting effect in EE due to saccharin after food intake. As these differences were progressively seen along time, we inferred that they were likely to be a chronic-adaptive mechanism rather than an acute effect simply determined by a single meal deprived of sugar. It is also reasonable to speculate that metabolism adjustments might have occurred in rats using saccharin due to long-term sugar restriction. Future studies, however, are needed to clarify this hypothesis.

We were not able to find similar studies comparing EE between saccharin and sucrose in experimental models in literature. In an interesting study of Swithers et al., Sprague-Dawley rats fed for a long time with artificialsweetened yogurt presented smaller increases in body temperature after an acute single standard meal compared with rats that were fed with glucose-sweetened yogurt during the same period. These authors observed a possible blunting thermal response to food in rats eating saccharin, suggesting that metabolic adjustments of energy expenditure could occur, which is completely in agreement to the data in the present study [9]. 


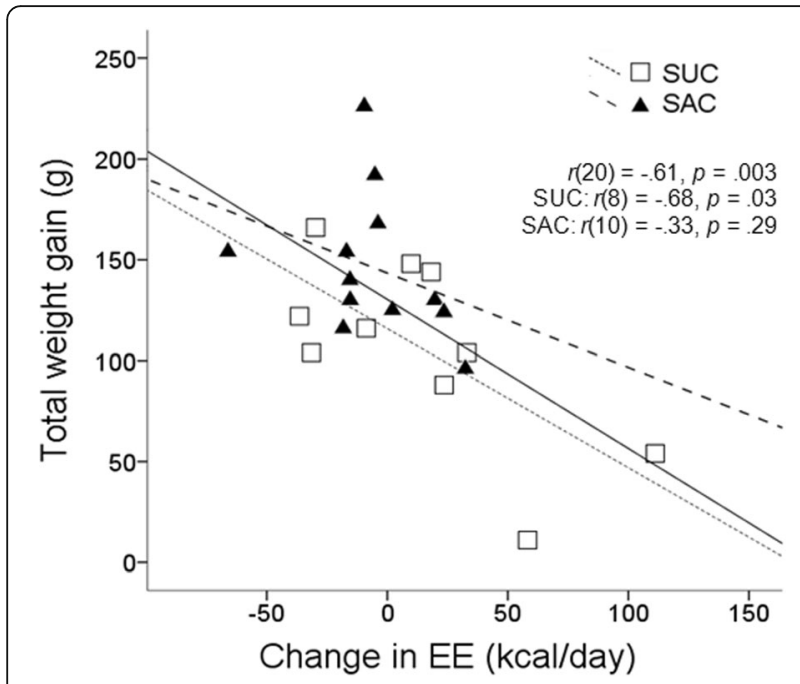

Fig. 4 Relationship between total weight gain ( $g$ ) and change in energy expenditure ( $\mathrm{kcal} /$ day) observed over the entire experiment demonstrated a significant and inverse correlation $[r(20)=-.61$, $p=.003]$ when analyzing both groups. In the stratified analysis we verified a similar correlation in the SUC group $[r(8)=-.68, p=.03]$ but not in the SAC group $[r(10)=-.33, p=.29]$

Plausible biological mechanisms for explaining EE differences between groups are speculative. During aerobic glucose oxidation, oxygen consumption usually rises proportionally to carbon dioxide production [15] and a parallel rise between $\mathrm{VO}_{2}$ and $\mathrm{VCO}_{2}$ curves is expected. Nevertheless, as in the present study, $\mathrm{VCO}_{2}$ production curve rose more steeply than the $\mathrm{VO}_{2}$ curve, suggesting that the anaerobic glycolysis could be the predominant metabolic pathway in SUC rats. This is supported by the fact that RER was near 1 in SUC group and near 0.7 in SAC group, suggesting that carbohydrates were the main fuel used by SUC rats, while fat was predominantly used by SAC rats.

This study has some important strengths. We conducted the experiment in the early post-absorptive state, which is important to minimize the animal stress and the ketogenesis due to the prolonged fasting state, which might interfere in results. It is also important that we measured carbon dioxide directly, and all respiratory variables were measured in duplicate, obtained in the following day, which considerably reduced EE determination variability. Finally, rats were kept in individual cages, which allowed accurate control of caloric intake. One potential limitation in this study was that the Weir formula was originally designed to calculate energy expenditure in humans, although it was later popularized for experimental research [14].

\section{Conclusion}

We concluded that long-term saccharin use may decrease energy expenditure at rest in the post-absorptive, period relative to sucrose in Wistar rats. On the other hand, long-term sucrose intake can increase energy expenditure in rats. This combined effect could explain, at least partially, the weight gain associated to saccharin in relation to sucrose in these animals.

\section{Abbreviations}

AUC: Area under curve; EE: Energy expenditure; FIPE: Fundo de Incentivo à Pesquisa; HCPA: Hospital de Clínicas de Porto Alegre; n.s.: Not significant; RER: Respiratory exchange ratio; SAC: Saccharin group; SE: Standard error; SP: São Paulo; SUC: Sucrose group; UFCSPA: Universidade Federal de Ciências da Saúde de Porto Alegre; UFRGS: Universidade Federal do Rio Grande do Sul; $\mathrm{VCO}_{2}$ : Volume of carbon dioxide produced; $\mathrm{VO}_{2}$ : Volume of oxygen consumed; vs: Versus; wk: Week

\section{Acknowledgements}

This study received financial support from the FIPE/HCPA. We are grateful to Jéssica Lorenzzi Elkfury and Pedro Ernesto Amaral da Silva by assistance in final experiments.

\section{Funding}

The main funding of this study was from HCPA (Hospital de Clínicas de Porto Alegre) through FIPE (Fundo de Incentivo à Pesquisa).

\section{Availability of data and materials}

Please contact author for data requests.

\section{Authors' contributions}

DEP participated in all phases of the study, including the conception, design, data collection, analysis and manuscript writing. KC participated in data collection, analysis interpretation of data and manuscript writing. RBN participated in design and data collection. PDL participated in the conception and study design. MCB Conceived the study idea and study design, data analysis, manuscript writing and provided funding for the study. All the authors agreed to be accountable for all aspects of the work in ensuring that questions related to the accuracy or integrity of any part of the work are appropriately investigated and resolved. All authors read and approved the final manuscript.

\section{Competing interest}

The authors declare not having any personal or financial support or involvement with organizations with financial interest in the subject matter or any actual or potential conflict of interest.

\section{Consent for publication}

All authors of this study are consistent with the publication of this article.

\section{Ethics approval}

This study was approved by ethics committee on animal research at UFSCPA and UFRGS. The protocol number at UFSCPA was $145 / 14$ and at UFRGS was 14-0018.

\section{Author details}

'Programa de Pós-Graduação em Medicina: Ciências Médicas, Universidade Federal do Rio Grande do Sul-UFRGS, Rua Ramiro Barcelos, n² 2400, CEP 90035-003, Bairro Rio Branco, Porto Alegre, RS, Brazil. ${ }^{2}$ Laboratório de Fisiologia Experimental, Universidade Federal de Ciências da Saúde de Porto Alegre-UFCSPA, Rua Sarmento Leite, n² 245, CEP 90050-170, Bairro Centro, Porto Alegre, RS, Brazil. ${ }^{3}$ Serviço de Medicina Interna, Hospital de Clínicas de Porto Alegre-Universidade Federal do Rio Grande do Sul, Rua Ramiro Barcelos, n² 2350, CEP 90035-903, Bairro Rio Branco, Porto Alegre, RS, Brazil.

Received: 21 September 2016 Accepted: 30 January 2017

Published online: 20 February 2017

\section{References}

1. Shankar P, Ahuja S, Sriram K. Non-nutritive sweeteners: review and update. Nutrition. 2013;29(11-12):1293-9. 
2. Colditz GA, Willett WC, Stampfer MJ, London SJ, Segal MR, Speizer FE. Patterns of weight change and their relation to diet in a cohort of healthy women. Am J Clin Nutr. 1990;51(6):1100-5.

3. Fowler SP, Williams K, Resendez RG, Hunt KJ, Hazuda HP, Stern MP. Fueling the obesity epidemic? Artificially sweetened beverage use and long-term weight gain. Obesity (Silver Spring). 2008;16(8):1894-900.

4. Dhingra R, Sullivan L, Jacques PF, Wang TJ, Fox CS, Meigs JB, et al. Soft drink consumption and risk of developing cardiometabolic risk factors and the metabolic syndrome in middle-aged adults in the community. Circulation. 2007;116(5):480-8.

5. Lutsey PL, Steffen LM, Stevens J. Dietary intake and the development of the metabolic syndrome: the Atherosclerosis Risk in Communities study. Circulation. 2008;117(6):754-61.

6. Davidson TL, Martin AA, Clark K, Swithers SE. Intake of high-intensity sweeteners alters the ability of sweet taste to signal caloric consequences: Implications for the learned control of energy and body weight regulation. Q J Exp Psychol (Colchester). 2011;64(7):1430-41.

7. Polyák E, Gombos K, Hajnal B, Bonyár-Müller K, Szabó S, GubicskóKisbenedek $A$, et al. Effects of artificial sweeteners on body weight, food and drink intake. Acta Physiol Hung. 2010;97(4):401-7.

8. Rogers PJ, Carlyle JA, Hill AJ, Blundell JE. Uncoupling sweet taste and calories: comparison of the effects of glucose and three intense sweeteners on hunger and food intake. Physiol Behav. 1988;43(5):547-52.

9. Swithers SE, Davidson TL. A role for sweet taste: calorie predictive relations in energy regulation by rats. Behav Neurosci. 2008;122(1):161-73.

10. Feijó FM, Ballard CR, Foletto KC, Batista BA, Neves AM, Ribeiro MF, et al. Saccharin and aspartame, compared with sucrose, induce greater weight gain in adult Wistar rats, at similar total caloric intake levels. Appetite. 2013;60(1):203-7.

11. Foletto KC, Melo Batista BA, Neves AM, de Matos FF, Ballard CR, Marques Ribeiro MF, et al. Sweet taste of saccharin induces weight gain without increasing caloric intake, not related to insulin-resistance in Wistar rats. Appetite. 2015;96:604-10

12. NRCC NRCCftUotGftCUoLA. The National Academies Collection: Reports funded by National Institutes of Health. Guide for the Care and Use of Laboratory Animals. 8th ed. Washington (DC): National Academies Press (US) National Academy of Sciences; 2011.

13. Rodrigues B, Figueroa DM, Mostarda CT, Heeren MV, Irigoyen MC, De Angelis K. Maximal exercise test is a useful method for physical capacity and oxygen consumption determination in streptozotocin-diabetic rats. Cardiovasc Diabetol. 2007;6:38.

14. Weir JB. New methods for calculating metabolic rate with special reference to protein metabolism. J Physiol. 1949;109(1-2):1-9.

15. Scott CB. Contribution of anaerobic energy expenditure to whole body thermogenesis. Nutr Metab. 2005;2:14.

\section{Submit your next manuscript to BioMed Central and we will help you at every step:}

- We accept pre-submission inquiries

- Our selector tool helps you to find the most relevant journal

- We provide round the clock customer support

- Convenient online submission

- Thorough peer review

- Inclusion in PubMed and all major indexing services

- Maximum visibility for your research

Submit your manuscript at www.biomedcentral.com/submit
Biomed Central 